\title{
Inference on the Parameters and Reliability Characteristics of the Generalized Inverse Weibull Distribution based on Records
}

\author{
Ajit Chaturvedi $^{1, a)}$ and Ananya Malhotra ${ }^{1, b)}$ \\ ${ }^{1}$ Department of Statistics, University of Delhi, Delhi - 110007, INDIA \\ a)ajitc2001@yahoo.co.in \\ ${ }^{\text {b)} C o r r e s p o n d i n g ~ a u t h o r: ~ m a l h o t r a . a n a n y a 3 @ g m a i l . c o m ~}$
}

\begin{abstract}
The inverse Weibull distribution has the ability to model failure rates which are quite common in reliability and biological studies. A three parameters generalized inverse Weibull distribution with decreasing and unimodal failure rate is studied. Two measures of reliability are discussed, namely $R(t)=P(X>t)$ and $P=P(X>Y)$. Point and interval estimation procedures are developed for the parameters, $R(t)$ and $P$ based on records. Two point estimators are developed, namely uniformly minimum variance unbiased estimators (UMVUE) and maximum likelihood estimators (MLE). A comparison of different methods of estimation is done through simulations and asymptotic confidence intervals of the parameters based on MLE and log transformed MLE are constructed. Confidence intervals for the MLE and UMVUE of the parametric functions are obtained. Testing procedures are also developed for various hypotheses.
\end{abstract}

\section{INTRODUCTION}

The reliability function $R(t)$ is defined as the probability of system survival until time $t$. Thus, if the random variable $(r v) X$ denotes the lifetime of an item or a system, then $R(t)=P(X>t)$. Another well known measure of reliability under stress-strength setup is the probability $P=P(X>Y)$, which represents the reliability of an item or a system of random strength $X$ subject to a random stress $Y$. In the literature, a lot of work has been done in the estimation and testing of $R(t)$ and $P$. For example, Bartholomew (1957, 1963), Pugh (1963), Basu (1964), Tong (1974, 1975), Johnson (1975), Kelley, Kelley and Schucany (1976), Sathe and Shah (1981), Chao (1982), Chaturvedi and Surinder (1999) developed inferential procedures for $R(t)$ and $P$ for exponential distribution. Constantine, Karson and Tse (1986) derived UMVUE and MLE of $P$ of the gamma distribution. Awad and Gharraf (1986) estimated $P$ of Burr distribution. For estimation of $R(t)$ of Maxwell and generalized Maxwell distributions, one may refer to Tyagi and Bhattacharya (1981) and Chaturvedi and Rani (1998), respectively. Inferences have been drawn for $R(t)$ and $P$ for some families of lifetime distributions by Chaturvedi and Rani (1997), Chaturvedi and Tomer (2003), Chaturvedi and Singh (2006, 2008), Chaturvedi and Kumari (2015) and Chaturvedi and Malhotra $(2016,2017)$. Chaturvedi and Tomer (2002) derived UMVUE of $R(t)$ and $P$ of negative binomial distribution. For exponentiated Weibull and Lomax distributions, the inferential procedures are available in Chaturvedi and Pathak (2012, 2013, 2014).

Chandler (1952) introduced the concept of record values. Based on records, inferential procedures for the parameters of different distributions have been developed by Glick (1978), Nagaraja (1988a,1988b), Balakrishan, Ahsanullah and Chan (1995), Arnold, Balakrishan and Nagaraja (1992), Habibi, Arghami and Ahmadi (2006), Arashi and Emadi (2008), Razmkhah and Ahmadi (2011), Belaghi, Arashi and Tabatabaey (2015) and others. To the best of our knowledge, no inferential procedures are available for the reliability functions of three parameter generalized inverse Weibull distribution based on record values. 
The rest of the paper is organized as follows. In Section 2, a three parameter generalized inverse Weibull distribution is discussed. In Section 3, we develop point estimation procedures based on record values when one parameter is unknown while the remaining are known and also discuss the case when all the parameters are unknown. As far as point estimation is concerned, we derive UMVUES and MLES. A new technique is developed for obtaining these estimators of $R(t)$ and $P$, in which initially the estimators of powers of parameter are obtained. These estimators are used to obtain the estimators of $R(t)$. Using the derivatives of the estimators of $R(t)$ with respect to time $t$, the estimators of sample probability density function at a specified point, are obtained which are further used to obtain the estimators of $P$. We also obtain expressions for the variance of the MLE and UMVUE of $R(t)$ which are used to compare the performance of these estimators of $R(t)$. In Section 4, asymptotic confidence intervals (CIs) for the parameters and reliability function are constructed. We also obtain confidence intervals based on log transformation of MLES of the parameter which have a better coverage probability. Confidence intervals for the MLES and UMVUES of the parametric functions are also obtained. In Section 5, testing procedures are developed for various hypotheses and finally, in Section 6, we present numerical findings.

\section{THE GENERALIZED INVERSE WEIBULL DISTRIBUTION}

The inverse Weibull distribution (IWD) has received some attention in the literature. Keller and Kamath (1982) studied the shapes of the density and failure rate functions for the basic inverse model. A $r v X$ follows IWD if its cumulative distribution function $(c d f)$ and probability distribution function $(p d f)$ take the following form respectively:

and

$$
G(x)=\exp \left(-\left(\frac{\alpha}{x}\right)^{\beta}\right) ; x>0, \alpha>0, \beta>0
$$

$$
g(x)=\beta \alpha^{\beta} x^{-(\beta+1)} \exp \left(-\left(\frac{\alpha}{x}\right)^{\beta}\right) ; x>0, \alpha>0, \beta>0 .
$$

The IWD is also a limiting distribution of the largest order statistic. Gusmão et al. (2009) introduced and studied a three parameter generalized inverse Weibull distribution (GIWD) whose $c d f$ is defined by elevating $G(x)$ to the power $\gamma>0$. Hence, the $p d f$ and $c d f$ of GIWD take the following form respectively:

$$
f(x ; \alpha, \beta, \gamma)=\gamma \beta \alpha^{\beta} x^{-(\beta+1)} \exp \left(-\gamma\left(\frac{\alpha}{x}\right)^{\beta}\right) ; x>0, \alpha, \beta, \gamma>0
$$

and

$$
F(x ; \alpha, \beta, \gamma)=\exp \left(-\gamma\left(\frac{\alpha}{x}\right)^{\beta}\right) ; x>0, \alpha, \beta, \gamma>0 .
$$

From equation (2), the reliability function at a specified time $t(>0)$ is

$$
R(t)=P(X>t)=1-\exp \left(-\gamma\left(\frac{\alpha}{t}\right)^{\beta}\right) .
$$

From equations (1) and (3), the hazard rate is given by

$$
h(t)=\gamma \beta \alpha^{\beta} t^{-(\beta+1)} \exp \left(-\gamma\left(\frac{\alpha}{t}\right)^{\beta}\right)\left[1-\exp \left(-\gamma\left(\frac{\alpha}{t}\right)^{\beta}\right)\right]^{-1} .
$$

It follows from equation (4) and Figure 1 that the hazard rate for every value of the parameters $\alpha, \beta$ and $\gamma$, initially increases with time and then decreases as time $t$ increases. 


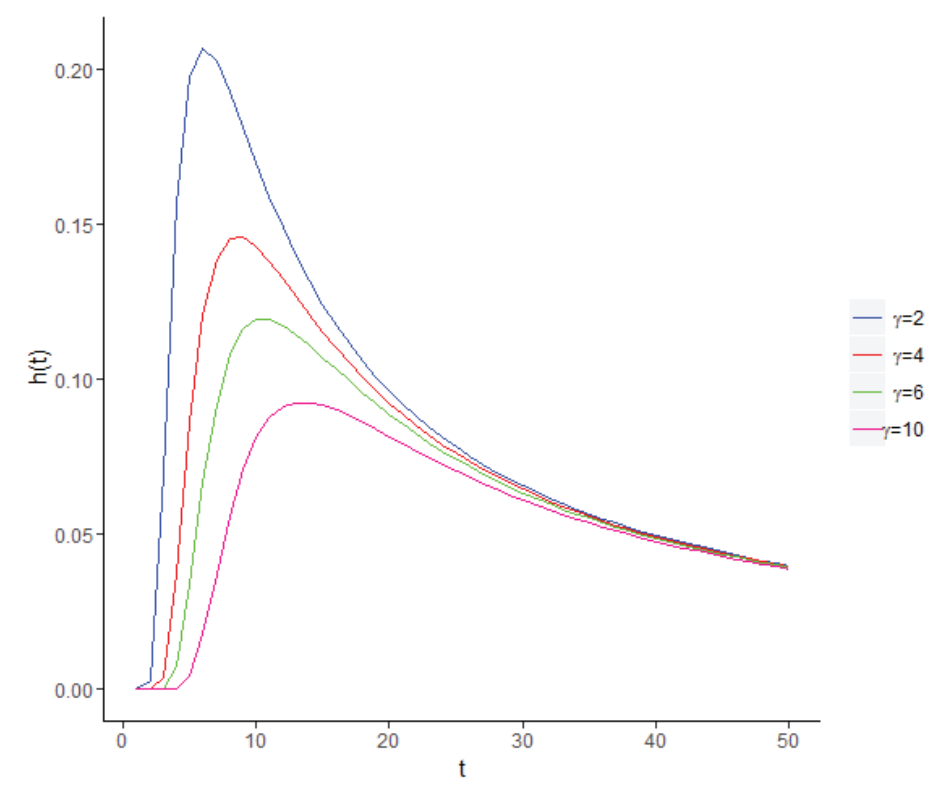

FIGURE 1. Hazard rate for several values of $\gamma$ where $\alpha=4$ and $\beta=2$.

\section{POINT ESTIMATION PROCEDURES}

Let $X_{1}, X_{2}, \ldots$ be an infinite sequence of independent and identically distributed (iid) rvs from equation (1). An observation $X_{j}$ will be called an upper record value (or simply a record) if its value exceeds all the previous observations. Thus $X_{j}$ is a record if $X_{j}>X_{i}$ for every $i<j$. The record time sequence $\left\{T_{n}, n \geq 0\right\}$ is defined as

The record value sequence $\left\{R_{n}\right\}$ is then given by

$$
\left\{\begin{array}{l}
T_{0}=1 \quad ; \text { with probability } 1 \\
T_{n}=\min \left\{j: X_{j}>X_{T_{n-1}}\right\} ; n \geq 1
\end{array}\right.
$$$$
R_{n}=X_{T_{n}} ; n=0,1,2, \ldots
$$

The likelihood function of $\gamma$ given the first $n+1$ upper record values $R_{0}, R_{1}, R_{2}, \ldots, R_{n}$ is

$$
L\left(\gamma \mid R_{0}, R_{1}, R_{2}, \ldots, R_{n}\right)=f\left(R_{n} ; \alpha, \beta, \gamma\right) \prod_{i=0}^{n-1} \frac{f\left(R_{i} ; \alpha, \beta, \gamma\right)}{1-F\left(R_{i} ; \alpha, \beta, \gamma\right)},
$$

where we assume that $\alpha$ and $\beta$ are known. It is easy to see that

$$
L\left(\gamma \mid R_{0}, R_{1}, R_{2}, \ldots, R_{n}\right)=\left(\gamma \beta \alpha^{\beta}\right)^{n+1} \exp \left(-\gamma\left(\frac{\alpha}{R_{n}}\right)^{\beta}\right) \prod_{i=0}^{n} R_{i}^{-(\beta+1)} .
$$

The following theorem provides UMVUE of powers of $\gamma$. This estimator will be utilized to obtain the UMVUE of reliability functions. For simplicity, we define

$$
U(x)=\left(\frac{\alpha}{x}\right)^{\beta}
$$

Theorem 1. For $q \in(-\infty, \infty), q \neq 0$, the UMVUE of $\gamma^{q}$ is given by

$$
\widetilde{\gamma^{q}}=\left\{\begin{aligned}
\frac{\Gamma(n+1)}{\Gamma(n-q+1)}\left(U\left(R_{n}\right)\right)^{-q} & ; n>q-1 \\
0 & ; \text { otherwise }
\end{aligned}\right.
$$

Proof: It follows from (3.1) and factorisation theorem [see Rohtagi and Saleh $\left(2012\right.$, p.361)] that $U\left(R_{n}\right)$ is a sufficient statistic for $\gamma$ and the $p d f$ of $U\left(R_{n}\right)$ is

$$
h\left(U\left(R_{n}\right) \mid \gamma\right)=\frac{\gamma^{n+1} U\left(R_{n}\right)^{n}}{\Gamma(n+1)} \exp \left(-\gamma U\left(R_{n}\right)\right)
$$


From equation (6), since the distribution of $U\left(R_{n}\right)$ belongs to the exponential family, it is also complete [see Rohtagi and Saleh (2012, p.367)]. The result now follows from (6) that

$$
E\left[U\left(R_{n}\right)^{-q}\right]=\frac{\Gamma(n-q+1)}{\Gamma(n+1)} \gamma^{q} .
$$

In the following theorem, we obtain UMVUE of the reliability function.

Theorem 2: The UMVUE of the reliability function is

Proof: It is easy to see that

$$
\tilde{R}(t)=\left\{\begin{aligned}
1-\left[1-\frac{U(t)}{U\left(R_{n}\right)}\right]^{n} ; & U(t)<U\left(R_{n}\right) . \\
0 ; & \text { otherwise }
\end{aligned}\right.
$$

$$
\begin{aligned}
R(t) & =1-\exp (-\gamma U(t)) \\
& =\sum_{i=0}^{\infty} \frac{(-1)^{i}}{i !}\{\gamma U(t)\}^{i} .
\end{aligned}
$$

Applying Theorem 1, it follows from (7) that

and the theorem follows.

$$
\tilde{R}(t)=1-\sum_{i=0}^{\infty} \frac{(-1)^{i}}{i !}\{U(t)\}^{i} \widetilde{\gamma}^{i}=1-\sum_{i=0}^{n}(-1)^{i}\left(\begin{array}{c}
n \\
i
\end{array}\right)\left\{\frac{U(t)}{U\left(R_{n}\right)}\right\}^{i}
$$
Theorem 2 .

The following corollary provides UMVUE of the sampled $p d f$. This estimator is derived with the help of

Corollary 1: The UMVUE of the sampled $p d f$ in (1) at a specified point $x$ is

$$
\tilde{f}(x ; \alpha, \beta, \gamma)=\left\{\begin{aligned}
\frac{n \beta \alpha^{\beta} x^{-(\beta+1)}}{U\left(R_{n}\right)}\left[1-\frac{U(x)}{U\left(R_{n}\right)}\right]^{n-1} & ; U(x)<U\left(R_{n}\right) . \\
0 & ; \text { otherwise }
\end{aligned}\right.
$$

Proof: We note that the expectation of $\int_{t}^{\infty} \tilde{f}(x ; \alpha, \beta, \gamma) d x$ with respect to $R_{n}$ is $R(t)$. Hence,

The result follows from Theorem 2 .

$$
\tilde{R}(t)=\int_{t}^{\infty} \tilde{f}(x ; \alpha, \beta, \gamma) d x .
$$
efficiency.

In the following theorem, we obtain expression for the variance of $\tilde{R}(t)$, which will be needed to study its

Theorem 3: The variance of $\tilde{R}(t)$ is given by

$$
\begin{aligned}
& \operatorname{Var}\{\tilde{R}(t)\}=\exp \{-\gamma U(t)\}\left[-2+\sum_{i=0}^{n} \frac{(\gamma U(t))^{i}}{i !}\right. \\
&+\frac{\{\gamma U(t)\}^{(n+1)}}{n !}\left[\frac{a_{n}}{\gamma U(t)}-a_{n-1} \exp \{\gamma U(t)\} E_{i}(-\gamma U(t))\right. \\
&+\sum_{i=0}^{n-2} a_{i}\left\{\sum_{m=1}^{n-i-1} \frac{(m-1) !}{(n-i-1) !}(-\gamma U(t))^{n-i-m-1}\right. \\
&\left.-\frac{1}{(n-i-1) !}(-\gamma U(t))^{n-i-1} \exp (\gamma U(t)) E_{i}(-\gamma U(t))\right\} \\
&\left.+\sum_{i=n+1}^{2 n} a_{i}(i-n) !\left(\frac{1}{\gamma U(t)}\right) \sum_{r=0}^{i-n+1} \frac{1}{r !}(\gamma U(t))^{r}\right]-[1-\exp \{-\gamma U(t)\}]^{2},
\end{aligned}
$$


where $a_{i}=(-1)^{i}\left(\begin{array}{c}2 n \\ i\end{array}\right)$ and $-E_{i}(-x)=\int_{x}^{\infty} \frac{e^{-u}}{u} d u$.

Proof: Using equation (6) and Theorem 2,

$$
\begin{aligned}
E\left\{\tilde{R}(t)^{2}\right\}= & \frac{\gamma^{n+1}}{\Gamma(n+1)} \int_{U(t)}^{\infty}\left[1-\left(1-\frac{U(t)}{U\left(R_{n}\right)}\right)^{n}\right]^{2}\left\{U\left(R_{n}\right)\right\}^{n} \exp \left\{-\gamma U\left(R_{n}\right)\right\} d U\left(R_{n}\right) \\
= & \frac{1}{\Gamma(n+1)}(\gamma U(t))^{n+1} \exp (-\gamma U(t)) \int_{0}^{\infty}(z+1)^{n} \exp (-z \gamma U(t)) d z \\
& +\frac{1}{\Gamma(n+1)}(\gamma U(t))^{n+1} \exp (-\gamma U(t)) \int_{0}^{\infty} \frac{z^{2 n}}{(1+z)^{n}} \exp (-z \gamma U(t)) d z \\
& -\frac{2}{\Gamma(n+1)}(\gamma U(t))^{n+1} \exp (-\gamma U(t)) \int_{0}^{\infty} z^{n} \exp (-z \gamma U(t)) d z \\
& =\exp (-\gamma U(t)) \sum_{i=0}^{n} \frac{(\gamma U(t))^{i}}{i !}+\frac{1}{\Gamma(n+1)}(\gamma U(t))^{n+1} \exp (-\gamma U(t)) I-2 \exp (-\gamma U(t))
\end{aligned}
$$

where

$$
I=\sum_{i=0}^{n} a_{i} \int_{0}^{\infty} \frac{1}{(z+1)^{n-i}} \exp (-z \gamma U(t)) d+\sum_{i=n+1}^{2 n} a_{i} \int_{0}^{\infty}(z+1)^{i-n} \exp (-z \gamma U(t)) d z
$$

Using a result of Erdélyi (1954)

we have

$$
\int_{0}^{\infty} \frac{\exp (-u p)}{(u+a)^{n}} d u=\sum_{m=1}^{n-1} \frac{(m-1) !(-p)^{n-m-1}}{(n-1) ! a^{m}}-\frac{(-p)^{n-1}}{(n-1) !} \exp (a p) E_{i}(-a p),
$$

$$
\begin{aligned}
& \int_{0}^{\infty} \frac{1}{(z+1)^{n-i}} \exp (-z \gamma U(t)) d z \\
&=\sum_{m=1}^{n-i-1} \frac{(m-1) !}{(n-i-1) !}(-\gamma U(t))^{n-i-m-1}-\frac{1}{(n-i-1) !}(-\gamma U(t))^{n-i-1} \exp (\gamma U(t)) E_{i}(-\gamma U(t)), \\
& i=0,1,2, \ldots n-2
\end{aligned}
$$

Furthermore,

$$
\begin{aligned}
\int_{0}^{\infty} \frac{1}{(1+z)} \exp (-z \gamma U(t)) d z & =\exp (\gamma U(t)) \int_{0}^{\infty} \frac{1}{z+1} \exp (-\gamma U(t)(z+1)) d z \\
& =-\exp (\gamma U(t)) E_{i}(-\gamma U(t)) .
\end{aligned}
$$

We have

$$
\int_{0}^{\infty} \exp (-z \gamma U(t)) d u=\left(\frac{1}{\gamma U(t)}\right)
$$

Finally, 


$$
\begin{aligned}
\int_{0}^{\infty}(1+z)^{i-n} \exp (-z \gamma U(t)) d z & =\sum_{r=0}^{i-n}\left(\begin{array}{c}
i-n \\
r
\end{array}\right) \int_{0}^{\infty} z^{i-n-r} \exp (1-z \gamma U(t)) d z \\
& =\sum_{r=0}^{i-n}\left(\begin{array}{c}
i-n \\
r
\end{array}\right)\left\{\frac{1}{\gamma U(t)}\right\}^{i-n-r+1} \Gamma(i-n-r+1)
\end{aligned}
$$
(9).

The theorem now follows on making substitutions from equations (11), (12), (13) and (14) in (10) and then using

Theorem 4: The MLE of $R(t)$ is given by

$$
\hat{R}(t)=1-\exp \left(\frac{-(n+1) U(t)}{U\left(R_{n}\right)}\right) .
$$

Proof: It can be easily seen from equation (5) that the MLE of $\gamma$ is $\hat{\gamma}=\frac{(n+1)}{U\left(R_{n}\right)}$. The theorem now follows from invariance property of MLE.

In the following corollary, we obtain the MLE of sampled $p d f$ with the help of Theorem 4 . This will be used to obtain MLE of $P$.

Corollary 2: The MLE of $f(x ; \alpha, \beta, \gamma)$ at a specified point $x$ is

$$
\hat{f}(x ; \alpha, \beta, \gamma)=\frac{(n+1) \beta \alpha^{\beta} x^{-(\beta+1)}}{U\left(R_{n}\right)} \exp \left(\frac{-(n+1) U(x)}{U\left(R_{n}\right)}\right) .
$$

Proof: The result follows from Theorem 4 on using the fact that

$$
\hat{f}(x ; \alpha, \beta, \gamma)=-\frac{d}{d t} \hat{R}(t)
$$

In the following theorem, we obtain the expression for variance of $\hat{R}(t)$.

Theorem 5: The variance of $\hat{R}(t)$ is given by

where $K_{r}(\cdot)$ is modified Bessel function of second kind of order $r$.

$$
\begin{aligned}
\operatorname{Var}(\hat{R}(t))=\frac{2}{n !} & \{2(n+1) \gamma U(t)\}^{\frac{n+1}{2}} K_{n+1}(2 \sqrt{2(n+1) \gamma U(t)}) \\
& -\left[\frac{2}{n !}\{(n+1) \gamma U(t)\}^{\frac{n+1}{2}} K_{n+1}(2 \sqrt{(n+1) \gamma U(t)})\right]^{2},
\end{aligned}
$$

Proof: Using equation (6) and Theorem 4, we have

$$
\begin{aligned}
E(\hat{R}(t)) & =\frac{\gamma^{n+1}}{\Gamma(n+1)} \int_{0}^{\infty}\left[1-\exp \left(-\frac{(n+1) U(t)}{U\left(R_{n}\right)}\right)\right] \exp \left(-\gamma U\left(R_{n}\right)\right)\left\{U\left(R_{n}\right)\right\}^{n} d U\left(R_{n}\right) \\
& =1-\frac{1}{\Gamma(n+1)} \int_{0}^{\infty} \exp \left[-\left\{y+\frac{(n+1) \gamma U(t)}{y}\right\}\right] y^{n} d y .
\end{aligned}
$$

Applying a result of Watson (1952)

$$
\int_{0}^{\infty} u^{-m} \exp \left\{-\left(a u+\frac{b}{u}\right)\right\} d u=2\left(\frac{a}{b}\right)^{\frac{m-1}{2}} K_{m-1}(2 \sqrt{a b})
$$

[it is to be noted that $K_{-m}(\cdot)=K_{m}(\cdot)$ for $\left.m=0,1,2, \ldots\right]$, we obtain from (15) that

$$
E(\hat{R}(t))=1-\frac{2}{n !}\{(n+1) \gamma U(t)\}^{\frac{n+1}{2}} K_{n+1}(2 \sqrt{(n+1) \gamma U(t)}) .
$$

Similarly, we can obtain the expression for $E\left(\hat{R}(t)^{2}\right)$ and the result follows.

Let $X$ and $Y$ be two independent rvs from generalized inverse Weibull distribution with $p d f f\left(x ; \alpha_{1}, \beta_{1}, \gamma_{1}\right)$ and $f\left(y ; \alpha_{2}, \beta_{2}, \gamma_{2}\right)$ respectively, i.e.

and

$$
f\left(x ; \alpha_{1}, \beta_{1}, \gamma_{1}\right)=\gamma_{1} \beta_{1} \alpha_{1}^{\beta_{1}} x^{-\left(\beta_{1}+1\right)} \exp \left\{-\gamma_{1}\left(\frac{\alpha_{1}}{x}\right)^{\beta_{1}}\right\} ; x>0, \alpha_{1}, \beta_{1}, \gamma_{1}>0
$$

$$
f\left(y ; \alpha_{2}, \beta_{2}, \gamma_{2}\right)=\gamma_{2} \beta_{2} \alpha_{2}^{\beta_{2}} y^{-\left(\beta_{2}+1\right)} \exp \left\{-\gamma_{2}\left(\frac{\alpha_{2}}{y}\right)^{\beta_{2}}\right\} ; y>0, \alpha_{2}, \beta_{2}, \gamma_{2}>0
$$


Let $\left\{R_{n}\right\}$ and $\left\{R_{m}^{*}\right\}$ be the record value sequences for $X^{\prime} S$ and $Y^{\prime} s$ respectively. For simplicity, we define $U(x)=\left(\frac{\alpha_{1}}{x}\right)^{\beta_{1}}$ and $V(y)=\left(\frac{\alpha_{2}}{y}\right)^{\beta_{2}}$.

Theorem 6: The UMVUE of $P$ is given by

$$
\tilde{P}=\left\{\begin{array}{c}
m \int_{0}^{\frac{V\left(R_{n}\right)}{V\left(R_{m}^{*}\right)}}(1-z)^{m-1}\left[1-\left\{1-U\left(R_{n}\right)^{-1}\left(\frac{\alpha_{1}}{\alpha_{2}}\right)^{\beta_{1}}\left(z V\left(R_{m}^{*}\right)\right)^{\frac{\beta_{1}}{\beta_{2}}}\right\}^{n}\right] d z ; R_{n}>R_{m}^{*} \\
m \int_{0}^{1}(1-z)^{m-1}\left[1-\left\{1-U\left(R_{n}\right)^{-1}\left(\frac{\alpha_{1}}{\alpha_{2}}\right)^{\beta_{1}}\left(z V\left(R_{m}^{*}\right)\right)^{\frac{\beta_{1}}{\beta_{2}}}\right\}^{n}\right] d z ; R_{n} \leq R_{m}^{*}
\end{array} .\right.
$$

Proof: It follows from Corollary 1 that the UMVUES of $f\left(x ; \alpha_{1}, \beta_{1}, \gamma_{1}\right)$ and $f\left(y ; \alpha_{2}, \beta_{2}, \gamma_{2}\right)$ at specified points $x$ and $y$ are respectively

$$
\tilde{f}\left(x ; \alpha_{1}, \beta_{1}, \gamma_{1}\right)=\left\{\begin{aligned}
\frac{n \beta_{1} \alpha_{1} \beta_{1} x^{-\left(\beta_{1}+1\right)}}{U\left(R_{n}\right)}\left[1-\frac{U(x)}{U\left(R_{n}\right)}\right]^{\mathrm{n}-1} & ; U(x)<U\left(R_{n}\right) \\
0 & ; \text { otherwise }
\end{aligned}\right.
$$

and

$$
\tilde{f}\left(y ; \alpha_{2}, \beta_{2}, \gamma_{2}\right)=\left\{\begin{array}{c}
\frac{n \beta_{2} \alpha_{2} \beta_{2} y^{-\left(\beta_{2}+1\right)}}{V\left(R_{m}^{*}\right)}\left[1-\frac{V(y)}{V\left(R_{m}^{*}\right)}\right]^{\mathrm{m}-1} ; V(y)<V\left(R_{m}^{*}\right) . \\
0 \quad \text { otherwise }
\end{array}\right.
$$

From the arguments similar to those used in the proof of Corollary 1,

$$
\begin{aligned}
\tilde{P} & =\int_{y=0}^{\infty} \int_{x=y}^{\infty} \tilde{f}\left(x ; \alpha_{1}, \beta_{1}, \gamma_{1}\right) \tilde{f}\left(y ; \alpha_{2}, \beta_{2}, \gamma_{2}\right) d x d y \\
& =\int_{y=0}^{\infty} \tilde{R}\left(y ; \alpha_{1}, \beta_{1}\right)\left\{-\frac{d}{d y} \tilde{R}\left(y ; \alpha_{2}, \beta_{2}\right)\right\} d y \\
& =m \int_{\max \left[\mathbb{Q}_{n}, R_{m}^{*}\right]}^{\infty}\left[1-\left\{1-\frac{U(y)}{U\left(R_{n}\right)}\right\}^{n}\right]\left\{\frac{m \beta_{2} \alpha_{2}^{\beta_{2}} y^{-\left(\beta_{2}+1\right)}}{V\left(R_{m}^{*}\right)}\right\}\left[1-\frac{V(y)}{V\left(R_{m}^{*}\right)}\right]^{m-1} d y
\end{aligned}
$$

The theorem now follows on considering the two cases and putting $\frac{V(y)}{V\left(R_{m}^{*}\right)}=z$.

Theorem 7: The MLE of $P$ is

Proof: We have,

$$
\hat{P}=\int_{0}^{\infty} e^{-z}\left[1-\exp \left(-(n+1) U\left(R_{n}\right)^{-1}\left(\frac{\alpha_{1}}{\alpha_{2}}\right)^{\beta_{1}}\left(\frac{z V\left(R_{m}^{*}\right)}{m+1}\right)^{\frac{\beta_{1}}{\beta_{2}}}\right)\right] d z .
$$

$$
\begin{aligned}
\hat{P} & =\int_{y=0}^{\infty} \int_{\substack{\infty=y \\
\infty}}^{\infty} \hat{f}\left(x ; \alpha_{1}, \beta_{1}, \gamma_{1}\right) \hat{f}\left(y ; \alpha_{2}, \beta_{2}, \gamma_{2}\right) d x d y \\
& =\int_{\substack{y=0 \\
\infty}} \hat{R}\left(y ; \alpha_{1}, \beta_{1}\right) \hat{f}\left(y ; \alpha_{2}, \beta_{2}, \gamma_{2}\right) d y \\
& =\int_{y=0}^{\infty}\left[1-\exp \left\{\frac{-(n+1) U(y)}{U\left(R_{n}\right)}\right\}\right]\left\{\frac{(m+1) \beta_{2} \alpha_{2} \beta_{2} y^{-\left(\beta_{2}+1\right)}}{V\left(R_{m}^{*}\right)}\right\} \exp \left\{\frac{-(m+1) V(y)}{V\left(R_{m}^{*}\right)}\right\} d y .
\end{aligned}
$$

The result now follows on putting $\left\{\frac{(m+1) V(y)}{V\left(R_{m}^{*}\right)}\right\}=z$.

Now we consider the case when all the parameters $\alpha, \beta$ and $\gamma$ are unknown. From (5),the log-likelihood function is given by 


$$
l(\alpha, \beta, \gamma)=(n+1) \log (\gamma)+(n+1) \log (\beta)+(n+1) \beta \log (\alpha)-\gamma\left(\frac{\alpha}{R_{n}}\right)^{\beta}-(\beta+1) \sum_{i=0}^{n} \log \left(R_{i}\right) .
$$

The MLES of $\alpha, \beta$ and $\gamma$ are the solutions of the three simultaneous equations given below

$$
\begin{gathered}
\frac{n+1}{\gamma}-\left(\frac{\alpha}{R_{n}}\right)^{\beta}=0 \\
\frac{(n+1)}{\beta}+(n+1) \log (\alpha)-\gamma\left(\frac{\alpha}{R_{n}}\right)^{\beta} \log \left(\frac{\alpha}{R_{n}}\right)-\sum_{i=0}^{n} \log \left(R_{i}\right)=0
\end{gathered}
$$

and

$$
\frac{(n+1) \beta}{\alpha}-\gamma \beta\left(\frac{\alpha^{\beta-1}}{R_{n}^{\beta}}\right)=0 .
$$

From (17) we get

$$
\hat{\gamma}=\frac{n+1}{\left(\frac{\hat{\alpha}}{R_{n}}\right)^{\widehat{\beta}}}
$$

where $\hat{\alpha}, \hat{\beta}$ and $\hat{\gamma}$ are the MLES of $\alpha, \beta$ and $\gamma$ respectively. Since these non-linear equations do not have a closed form solution, therefore we apply Newton Raphson algorithm to compute MLES of $\alpha$ and $\beta$. These values of MILESnd $\beta$ so obtained can be substituted in (20) to obtain MLE of $\gamma$. It is to be noted that from Theorem 4, Theorem 7 and invariance property of MLE that the MLE of $R(t)$ is

$$
\hat{R}(t)=\exp \left\{\frac{-(n+1) U(t)}{U\left(R_{n}\right)}\right\},
$$

where $U(x)=\left(\frac{\widehat{\alpha}}{x}\right)^{\widehat{\beta}}$ whereas the MLE of $P$ is given by

where $U(x)=\left(\frac{\widehat{\frac{\alpha_{1}}{x}}}{x}\right)^{\widehat{\beta_{1}}}, V(x)=\left(\frac{\widehat{\frac{\alpha_{2}}{x}}}{{ }^{\widehat{\beta_{2}}}}\right.$.

$$
\hat{P}=\int_{0}^{\infty} e^{-z}\left[1-\exp \left(-(n+1) U\left(R_{n}\right)^{-1}\left(\frac{\widehat{\alpha_{1}}}{\widehat{\alpha_{2}}}\right)^{\widehat{\beta_{1}}}\left(\frac{z V\left(R_{m}^{*}\right)}{m+1}\right)^{\frac{\widehat{\beta_{1}}}{\bar{\beta}_{2}}}\right)\right] d z
$$

\section{CONFIDENCE INTERVALS}

The Fisher information matrix of $\theta=(\alpha, \beta, \gamma)^{T}$ is

where

$$
I(\theta)=-E\left[\begin{array}{ccc}
\frac{\partial^{2} l}{\partial \alpha^{2}} & \frac{\partial^{2} l}{\partial \alpha \partial \beta} & \frac{\partial^{2} l}{\partial \alpha \partial \gamma} \\
\frac{\partial^{2} l}{\partial \beta \partial \alpha} & \frac{\partial^{2} l}{\partial \beta^{2}} & \frac{\partial^{2} l}{\partial \beta \partial \gamma} \\
\frac{\partial^{2} l}{\partial \gamma \partial \alpha} & \frac{\partial^{2} l}{\partial \gamma \partial \beta} & \frac{\partial^{2} l}{\partial \gamma^{2}}
\end{array}\right]
$$

$$
\begin{gathered}
\frac{\partial^{2} l}{\partial \gamma^{2}}=\frac{-(n+1)}{\gamma^{2}}, \\
\frac{\partial^{2} l}{\partial \beta \partial \gamma}=\frac{\partial^{2} l}{\partial \gamma \partial \beta}=-\left(\frac{\alpha}{R_{n}}\right)^{\beta} \log \left(\frac{\alpha}{R_{n}}\right), \frac{\partial^{2} l}{\partial \alpha \partial \gamma}=\frac{\partial^{2} l}{\partial \gamma \partial \alpha}=\frac{-\beta \alpha^{\beta-1}}{R_{n} \beta},
\end{gathered}
$$




$$
\begin{gathered}
\frac{\partial^{2} l}{\partial \alpha \partial \beta}=\frac{\partial^{2} l}{\partial \beta \partial \alpha}=\frac{n+1}{\alpha}-\frac{\gamma \alpha^{\beta-1}}{R_{n}{ }^{\beta}}\left\{1+\beta \log \left(\frac{\alpha}{R_{n}}\right)\right\}, \\
\frac{\partial^{2} l}{\partial \alpha^{2}}=-\frac{\beta(n+1)}{\alpha^{2}}-\frac{\gamma \beta(\beta-1) \alpha^{\beta-2}}{R_{n}^{\beta}}
\end{gathered}
$$

and

$$
\frac{\partial^{2} l}{\partial \beta^{2}}=\frac{-(n+1)}{\beta^{2}}-\gamma\left(\frac{\alpha}{R_{n}}\right)^{\beta}\left(\log \left(\frac{\alpha}{R_{n}}\right)\right)^{2} .
$$

Since it is a complicated task to obtain the expectation of the above expressions, therefore we use observed Fisher information matrix which is obtained by dropping the expectation sign. The asymptotic variance-covariance matrix of the MLES is the inverse of $I(\hat{\theta})$. After obtaining the inverse matrix, we get variance of $\hat{\alpha}, \hat{\beta}$ and $\hat{\gamma}$. We use these values to construct confidence intervals (CIs) of $\alpha, \beta$ and $\gamma$ respectively. Assuming asymptotic normality of the MLES, CIs for $\alpha, \beta$ and $\gamma$ are constructed. Let $\hat{\sigma}^{2}(\hat{\alpha}), \hat{\sigma}^{2}(\hat{\beta})$ and $\hat{\sigma}^{2}(\hat{\gamma})$ be the estimated variances of $\hat{\alpha}, \hat{\beta}$ and $\hat{\gamma}$ respectively. Then $100(1-\varepsilon) \%$ asymptotic CIs for $\alpha, \beta$ and $\gamma$ are respectively given by

$$
\left(\hat{\alpha}-Z_{\frac{\varepsilon}{2}} \hat{\sigma}(\hat{\alpha}), \hat{\alpha}+Z_{\frac{\varepsilon}{2}} \hat{\sigma}(\hat{\alpha})\right),\left(\hat{\beta}-Z_{\frac{\varepsilon}{2}} \hat{\sigma}(\hat{\beta}), \hat{\beta}+Z_{\frac{\varepsilon}{2}} \hat{\sigma}(\hat{\beta})\right) \text { and }\left(\hat{\gamma}-Z_{\frac{\varepsilon}{2}} \hat{\sigma}(\hat{\gamma}), \hat{\gamma}+Z_{\frac{\varepsilon}{2}} \hat{\sigma}(\hat{\gamma})\right)
$$

where $Z_{\frac{\varepsilon}{2}}$ is the upper $100(1-\varepsilon)$ percentile point of standard normal distribution. Using these CIs, one can easily obtain the $100(1-\varepsilon) \%$ asymptotic $\mathrm{CI}$ for $R(t)$ as follows:

$$
\left(1-\exp \left(-\left(\hat{\gamma}+Z_{\frac{\varepsilon}{2}} \hat{\sigma}(\hat{\gamma})\right)\left(\frac{\hat{\alpha}+Z_{\frac{\varepsilon}{2}} \hat{\sigma}(\hat{\alpha})}{t}\right)^{\widehat{\beta}+Z_{\varepsilon} \hat{\sigma}(\widehat{\beta})}\right), 1-\exp \left(-\left(\hat{\gamma}-Z_{\frac{\varepsilon}{2}} \hat{\sigma}(\hat{\gamma})\right)\left(\frac{\hat{\alpha}-Z_{\frac{\varepsilon}{2}} \hat{\sigma}(\hat{\alpha})}{t}\right)^{\widehat{\beta}-Z_{\varepsilon} \widehat{\sigma}(\widehat{\beta})}\right)\right)
$$

Meeker and Escober (1998) reported that the asymptotic CI based on log transformed MLE has better coverage probability. An approximate $100(1-\varepsilon) \%$ CIs for $\log (\alpha), \log (\beta)$ and $\log (\gamma)$ are

$$
\begin{array}{r}
\left(\log (\hat{\alpha})-Z_{\frac{\varepsilon}{2}} \hat{\sigma}(\log (\hat{\alpha})), \log (\hat{\alpha})+Z_{\frac{\varepsilon}{2}} \hat{\sigma}(\log (\hat{\alpha}))\right), \\
\left(\log (\hat{\beta})-Z_{\frac{\varepsilon}{2}} \hat{\sigma}(\log (\hat{\beta})), \log (\hat{\beta})+Z_{\frac{\varepsilon}{2}} \hat{\sigma}(\log (\hat{\beta}))\right)
\end{array}
$$

and

$$
\left(\log (\hat{\gamma})-Z_{\frac{\varepsilon}{2}} \hat{\sigma}(\log (\hat{\gamma})), \log (\hat{\gamma})+Z_{\frac{\varepsilon}{2}} \hat{\sigma}(\log (\hat{\gamma}))\right) .
$$

where $\hat{\sigma}^{2}(\log (\hat{\alpha}))$ is the estimated variance of $\log (\alpha)$ and is approximated by $\hat{\sigma}^{2}(\log (\hat{\alpha}))=\frac{\widehat{\sigma}^{2}(\widehat{\alpha})}{\widehat{\alpha}^{2}}$. Similarly, $\hat{\sigma}^{2}(\log (\hat{\beta}))$ and $\hat{\sigma}^{2}(\log (\hat{\gamma}))$ are the estimated variance of $\log (\beta)$ and $\log (\gamma)$ and are approximated by $\hat{\sigma}^{2}(\log (\hat{\beta}))=\frac{\widehat{\sigma}^{2}(\widehat{\beta})}{\widehat{\beta}^{2}}$ and $\hat{\sigma}^{2}(\log (\hat{\gamma}))=\frac{\widehat{\sigma}^{2}(\widehat{\gamma})}{\widehat{\gamma}^{2}}$ respectively. Hence, approximate $100(1-\varepsilon) \%$ CI for $\alpha, \beta$ and $\gamma$ are

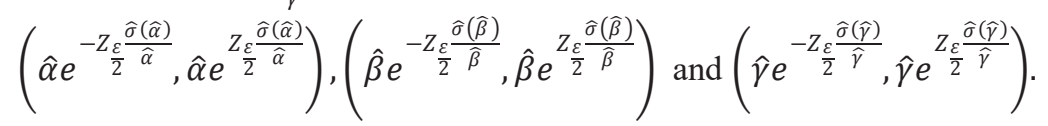

Now, we construct interval estimates of UMVUE and MLE of $\gamma$. From (6) it follows that $2 \gamma\left(\frac{\alpha}{R_{n}}\right)^{\beta} \sim \chi_{2(n+1)}^{2}$. Thus $100(1-\varepsilon) \%$ CI for $\tilde{\gamma}$ and $\hat{\gamma}$ are respectively obtained as

$$
\left(\frac{2 \gamma n}{\chi_{2(n+1)}^{2}\left(1-\frac{\varepsilon}{2}\right)}, \frac{2 \gamma n}{\chi_{2(n+1)}^{2}\left(\frac{\varepsilon}{2}\right)}\right) \text { and }\left(\frac{2 \gamma(n+1)}{\chi_{2(n+1)}^{2}\left(1-\frac{\varepsilon}{2}\right)}, \frac{2 \gamma(n+1)}{\chi_{2(n+1)}^{2}\left(\frac{\varepsilon}{2}\right)}\right) \text {. }
$$

Similarly, we construct interval estimates of UMVUE and MLE of $R(t)$. Thus $100(1-\varepsilon) \%$ CI for $\tilde{R}(t)$ and $\hat{R}(t)$ are respectively obtained as 
and

$$
\left(1-\left[1-\frac{2 \gamma U(t)}{\chi_{2(n+1)}^{2}\left(1-\frac{\varepsilon}{2}\right)}\right]^{n}, 1-\left[1-\frac{2 \gamma U(t)}{\chi_{2(n+1)}^{2}\left(\frac{\varepsilon}{2}\right)}\right]^{n}\right)
$$

$$
\left(1-\exp \left(-\frac{2 \gamma(n+1) U(t)}{\chi_{2(n+1)}^{2}\left(1-\frac{\varepsilon}{2}\right)}\right), 1-\exp \left(-\frac{2 \gamma(n+1) U(t)}{\chi_{2(n+1)}^{2}\left(\frac{\varepsilon}{2}\right)}\right)\right)
$$

\section{TESTING OF HYPOTHESES}

For known $\alpha$ and $\beta$, suppose we have to test the hypothesis $H_{\mathrm{o}}: \gamma=\gamma_{\mathrm{o}}$ against $H_{1}: \gamma \neq \gamma_{\mathrm{o}}$. It follows from (5) that, under $H_{\mathrm{o}}$,

and

$$
\sup _{\Theta_{\mathrm{o}}} L\left(\gamma \mid R_{0}, R_{1}, \ldots, R_{n}\right)=\left(\gamma_{o} \beta \alpha^{\beta}\right)^{n+1} \exp \left\{-\gamma_{o}\left(\frac{\alpha}{R_{n}}\right)^{\beta}\right\} \prod_{i=0}^{n} R_{i}^{-(\beta+1)} ; \Theta_{\mathrm{o}}=\left\{\gamma: \gamma=\gamma_{\mathrm{o}}\right\}
$$

$$
\sup _{\Theta} L\left(\gamma \mid R_{0}, R_{1}, \ldots, R_{n}\right)=\left\{\frac{(n+1) \beta \alpha^{\beta}}{\left(\frac{\alpha}{R_{n}}\right)^{\beta}}\right\}^{n+1} \exp (-(n+1)) \prod_{i=0}^{n} R_{i}^{-(\beta+1)} ; \Theta=\{\gamma: \gamma>0\} .
$$

Therefore, the likelihood ratio (LR) is given by

$$
\begin{aligned}
\emptyset\left(R_{0}, R_{1}, \ldots, R_{n}\right) & =\frac{\sup _{\Theta_{o}} L\left(\gamma \mid R_{0}, R_{1}, \ldots, R_{n}\right)}{\sup _{\Theta} L\left(\gamma \mid R_{0}, R_{1}, \ldots, R_{n}\right)} \\
& =\left\{\frac{\gamma_{o}\left(\frac{\alpha}{R_{n}}\right)^{\beta}}{n+1}\right\}^{n+1} \exp \left\{-\gamma_{o}\left(\frac{\alpha}{R_{n}}\right)^{\beta}+(n+1)\right\}
\end{aligned}
$$

We note that the first term on the right hand side of (21) is monotonically increasing and the second term is monotonically decreasing in $\left(\frac{\alpha}{R_{n}}\right)^{\beta}$. It follows from (6) that $2 \gamma_{\mathrm{o}}\left(\frac{\alpha}{R_{n}}\right)^{\beta} \sim \chi_{2(n+1)}^{2}$. Thus, the critical region is given by

$$
\left\{0<\left(\frac{\alpha}{R_{n}}\right)^{\beta}<l_{\mathrm{o}}\right\} \cup\left\{l_{\mathrm{o}}{ }^{\prime}<\left(\frac{\alpha}{R_{n}}\right)^{\beta}<\infty\right\},
$$

where $l_{\mathrm{o}}$ and $l_{\mathrm{o}}{ }^{\prime}$ are obtained such that $l_{\mathrm{o}}=\frac{\chi_{2(n+1)}^{2}\left(\frac{\varepsilon}{2}\right)}{2 \gamma_{\mathrm{o}}}$ and $l_{\mathrm{o}}{ }^{\prime}=\frac{\chi_{2(n+1)}^{2}\left(1-\frac{\varepsilon}{2}\right)}{2 \gamma_{\mathrm{o}}}$.

An important hypothesis in life-testing experiments is $H_{\mathrm{o}}: \gamma \leq \gamma_{\mathrm{o}}$ against $H_{1}: \gamma>\gamma_{\mathrm{o}}$. It follows from (5) that for $\gamma_{1}>\gamma_{2}$,

$$
\frac{L\left(\gamma_{1} \mid R_{0}, R_{1}, \ldots, R_{n}\right)}{L\left(\gamma_{2} \mid R_{0}, R_{1}, \ldots, R_{n}\right)}=\left(\frac{\gamma_{1}}{\gamma_{2}}\right)^{n+1} \exp \left\{\left(\gamma_{2}-\gamma_{1}\right)\left(\frac{\alpha}{R_{n}}\right)^{\beta}\right\}
$$

It follows from (22) that $f(x ; \alpha, \beta, \gamma)$ has monotone likelihood ratio in $\left(\frac{\alpha}{R_{n}}\right)^{\beta}$. Thus, the uniformly most powerful critical region for testing $H_{\mathrm{o}}$ against $H_{1}$ is given by [see Lehmann $(1959, \mathrm{p} .88)$ ]

where $l_{\mathrm{o}}{ }^{\prime \prime}=\frac{\chi_{2(n+1)}^{2}(\varepsilon)}{2 \gamma_{o}}$

$$
\emptyset\left(R_{0}, R_{1}, \ldots, R_{n}\right)=\left\{\begin{array}{cc}
1 & ;\left(\frac{\alpha}{R_{n}}\right)^{\beta} \leq l_{\mathrm{o}}^{\prime \prime} \\
0 \quad ; \text { otherwise }
\end{array}\right.
$$




\section{NUMERICAL FINDINGS}

In this section we use Monte Carlo simulation techinque to obtain estimates under this scheme. It involves the following steps:

1. For known values of $\alpha_{1}, \beta_{1}, \gamma_{1}, \alpha_{2}, \beta_{2}$ and $\gamma_{2}$, we generate 1000 samples each from distribution of $X \sim \operatorname{Gamma}\left(n+1, \gamma_{1}\right)$ and $Y \sim \operatorname{Gamma}\left(m+1, \gamma_{2}\right)$ for specified values of $n$ and $m$ to obtain $X_{j}$ and $Y_{j}, j=$ $1,2, \ldots, 1000$, respectively.

2. Compute $U\left(R_{n}\right)=\frac{1}{1000} \sum_{j=1}^{1000} X_{j}$ and $V\left(R_{m}^{*}\right)=\frac{1}{1000} \sum_{j=1}^{1000} Y_{j}$.

For specified value of $t$, compute $R(t)$ for $\operatorname{GIWD}\left(\alpha_{1}, \beta_{1}, \gamma_{1}\right)$ and hence compute MLE and UMVUE of $R(t)$. It can be easily shown that $P=1-\gamma_{2} \beta_{2} \alpha_{2}^{\beta_{2}} \int_{y=0}^{\infty} y^{-\left(\beta_{2}+1\right)} \exp \left(-\gamma_{1}\left(\frac{\alpha_{1}}{y}\right)^{\beta_{1}}-\gamma_{2}\left(\frac{\alpha_{2}}{y}\right)^{\beta_{2}}\right) d y$.

In Table 1 , for $\alpha_{1}=2, \beta_{1}=3, \gamma_{1}=0.5, \alpha_{2}=5, \beta_{2}=2$ and $\gamma_{2}=4$, we have shown MLE and UMVUE of $\gamma_{1}$ for several values of $n$. For $t=5, R(t)=0.0314$ and the MLE and UMVUE of $R(t)$ are shown in Table 1 for several values of $n . \quad P=0.00527$ by the above expression and the MLE and UMVUE of $P$ are shown in Table 1 for several values of $n$ and $m$.

TABLE 1. MLE and UMVUE of $\gamma, R(t)$ and $P$.

\begin{tabular}{ccccccc}
\hline$n, m$ & $\hat{\gamma}$ & $\tilde{\gamma}$ & $\hat{R}(t)$ & $\tilde{R}(t)$ & $\hat{P}$ & $\tilde{P}$ \\
\hline 10,10 & 0.50252 & 0.45684 & 0.03165 & 0.02886 & 0.00535 & 0.00472 \\
10,20 & 0.50010 & 0.45463 & 0.03150 & 0.02872 & 0.00521 & 0.00467 \\
10,50 & 0.50062 & 0.45511 & 0.03153 & 0.02875 & 0.00532 & 0.00481 \\
10,100 & 0.49313 & 0.44831 & 0.03107 & 0.02832 & 0.00519 & 0.00471 \\
20,10 & 0.49988 & 0.47608 & 0.03149 & 0.03003 & 0.00533 & 0.00492 \\
20,20 & 0.49972 & 0.47593 & 0.03148 & 0.03002 & 0.00533 & 0.00500 \\
20,50 & 0.49929 & 0.47552 & 0.03145 & 0.03000 & 0.00526 & 0.00498 \\
20,100 & 0.50727 & 0.48312 & 0.03194 & 0.03047 & 0.00538 & 0.00511 \\
50,10 & 0.50045 & 0.49064 & 0.03152 & 0.03092 & 0.00523 & 0.00497 \\
50,20 & 0.49793 & 0.48817 & 0.03137 & 0.03077 & 0.00521 & 0.00503 \\
50,50 & 0.49764 & 0.48789 & 0.03135 & 0.03075 & 0.00518 & 0.00505 \\
50,100 & 0.50067 & 0.49086 & 0.03154 & 0.03094 & 0.00528 & 0.00516 \\
100,10 & 0.50021 & 0.49526 & 0.03151 & 0.03120 & 0.00532 & 0.00510 \\
100,20 & 0.50104 & 0.49608 & 0.03156 & 0.03126 & 0.00534 & 0.00520 \\
100,50 & 0.50149 & 0.49653 & 0.03159 & 0.03128 & 0.00529 & 0.00520 \\
100,100 & 0.49728 & 0.49237 & 0.03133 & 0.03102 & 0.00522 & 0.00515 \\
\hline
\end{tabular}

In order to investigate the performance of the estimators obtained under this scheme, we have evaluated $\operatorname{Var}(\tilde{R}(t))$ and $\operatorname{MSE}(\hat{R}(t))$ for $\alpha=2, \beta=5$ and $\gamma=3$. Table 2 shows $\operatorname{Var}(\tilde{R}(t))$ and $\operatorname{MSE}(\hat{R}(t))$ for $t=$ $5(1) 30$ and $n=5,10,20$ and 40. Figure 2 compares the variance UMVUE of reliability function with the mean square error of MLE of reliability function calculated in Table 2 as time $t$ increases for $n=20$. 
TABLE 2. Mean Square Error of MLE and UMVUE of Reliability function.

\begin{tabular}{|c|c|c|c|c|c|c|c|c|}
\hline \multirow{2}{*}{$\begin{array}{r}n \\
t^{n} \\
\end{array}$} & \multicolumn{2}{|c|}{5} & \multicolumn{2}{|c|}{10} & \multicolumn{2}{|c|}{20} & \multicolumn{2}{|c|}{40} \\
\hline & $\operatorname{Var}(\tilde{R}(t))$ & $\operatorname{MSE}(\hat{R}(t))$ & $\operatorname{Var}(\tilde{R}(t))$ & $\operatorname{MSE}(\hat{R}(t))$ & $\operatorname{Var}(\tilde{R}(t))$ & $\operatorname{MSE}(\hat{R}(t))$ & $\operatorname{Var}(\tilde{R}(t))$ & $\operatorname{MSE}(\hat{R}(t))$ \\
\hline 5 & $2.17 \mathrm{E}-04$ & 0.84550 & $9.79 \mathrm{E}-05$ & 0.88259 & 4.66E-05 & 0.87962 & 2.27E-05 & 0.86917 \\
\hline 6 & $3.69 \mathrm{E}-05$ & 0.93542 & $1.65 \mathrm{E}-05$ & 0.95143 & 7.82E-06 & 0.95021 & $3.81 \mathrm{E}-06$ & 0.94576 \\
\hline 7 & 8.03E-06 & 0.96969 & $3.58 \mathrm{E}-06$ & 0.97729 & $1.70 \mathrm{E}-06$ & 0.97672 & $8.27 \mathrm{E}-07$ & 0.97462 \\
\hline 8 & $2.13 \mathrm{E}-06$ & 0.98436 & $9.47 \mathrm{E}-07$ & 0.98830 & 4.49E-07 & 0.98801 & 2.19E-07 & 0.98692 \\
\hline 9 & $6.58 \mathrm{E}-07$ & 0.99130 & 2.93E-07 & 0.99350 & $1.39 \mathrm{E}-07$ & 0.99333 & $6.75 \mathrm{E}-08$ & 0.99273 \\
\hline 10 & $2.30 \mathrm{E}-07$ & 0.99485 & $1.02 \mathrm{E}-07$ & 0.99616 & 4.84E-08 & 0.99606 & $2.36 \mathrm{E}-08$ & 0.99570 \\
\hline 11 & 8.87E-08 & 0.99680 & 3.94E-08 & 0.99761 & $1.87 \mathrm{E}-08$ & 0.99755 & $9.10 \mathrm{E}-09$ & 0.99733 \\
\hline 12 & $3.72 \mathrm{E}-08$ & 0.99793 & $1.65 \mathrm{E}-08$ & 0.99845 & 7.83E-09 & 0.99841 & $3.81 \mathrm{E}-09$ & 0.99827 \\
\hline 13 & $1.67 \mathrm{E}-08$ & 0.99861 & 7.42E-09 & 0.99896 & 3.52E-09 & 0.99894 & $1.71 \mathrm{E}-09$ & 0.99884 \\
\hline 14 & 7.96E-09 & 0.99904 & $3.54 \mathrm{E}-09$ & 0.99928 & $1.68 \mathrm{E}-09$ & 0.99927 & $8.17 \mathrm{E}-10$ & 0.99920 \\
\hline 15 & 3.99E-09 & 0.99932 & 1.78E-09 & 0.99949 & $8.41 \mathrm{E}-10$ & 0.99948 & $4.10 \mathrm{E}-10$ & 0.99943 \\
\hline 16 & 2.09E-09 & 0.99951 & $9.31 \mathrm{E}-10$ & 0.99963 & 4.41E-10 & 0.99962 & $2.15 \mathrm{E}-10$ & 0.99959 \\
\hline 17 & $1.14 \mathrm{E}-09$ & 0.99964 & $5.08 \mathrm{E}-10$ & 0.99973 & $2.41 \mathrm{E}-10$ & 0.99972 & $1.17 \mathrm{E}-10$ & 0.99970 \\
\hline 18 & $6.45 \mathrm{E}-10$ & 0.99973 & $2.87 \mathrm{E}-10$ & 0.99980 & $1.36 \mathrm{E}-10$ & 0.99979 & $6.62 \mathrm{E}-11$ & 0.99977 \\
\hline 19 & $3.76 \mathrm{E}-10$ & 0.99979 & $1.67 \mathrm{E}-10$ & 0.99984 & 7.91E-11 & 0.99984 & $3.85 \mathrm{E}-11$ & 0.99983 \\
\hline 20 & $2.25 \mathrm{E}-10$ & 0.99984 & $1.00 \mathrm{E}-10$ & 0.99988 & $4.74 \mathrm{E}-11$ & 0.99988 & $2.31 \mathrm{E}-11$ & 0.99987 \\
\hline 21 & $1.38 \mathrm{E}-10$ & 0.99987 & $6.14 \mathrm{E}-11$ & 0.99991 & 2.91E-11 & 0.99990 & $1.42 \mathrm{E}-11$ & 0.99989 \\
\hline 22 & $8.67 \mathrm{E}-11$ & 0.99990 & $3.86 \mathrm{E}-11$ & 0.99993 & $1.83 \mathrm{E}-11$ & 0.99992 & $8.90 \mathrm{E}-12$ & 0.99992 \\
\hline 23 & $5.56 \mathrm{E}-11$ & 0.99992 & $2.47 \mathrm{E}-11$ & 0.99994 & $1.17 \mathrm{E}-11$ & 0.99994 & $5.70 \mathrm{E}-12$ & 0.99993 \\
\hline 24 & $3.63 \mathrm{E}-11$ & 0.99994 & $1.61 \mathrm{E}-11$ & 0.99995 & $7.65 \mathrm{E}-12$ & 0.99995 & $3.73 \mathrm{E}-12$ & 0.99995 \\
\hline 25 & $2.42 \mathrm{E}-11$ & 0.99995 & $1.07 \mathrm{E}-11$ & 0.99996 & $5.09 \mathrm{E}-12$ & 0.99996 & $2.48 \mathrm{E}-12$ & 0.99996 \\
\hline 26 & $1.63 \mathrm{E}-11$ & 0.99996 & $7.25 \mathrm{E}-12$ & 0.99997 & $3.44 \mathrm{E}-12$ & 0.99997 & $1.67 \mathrm{E}-12$ & 0.99996 \\
\hline 27 & $1.12 \mathrm{E}-11$ & 0.99996 & 4.97E-12 & 0.99997 & $2.36 \mathrm{E}-12$ & 0.99997 & $1.15 \mathrm{E}-12$ & 0.99997 \\
\hline 28 & $7.78 \mathrm{E}-12$ & 0.99997 & $3.46 \mathrm{E}-12$ & 0.99998 & $1.64 \mathrm{E}-12$ & 0.99998 & $7.98 \mathrm{E}-13$ & 0.99998 \\
\hline 29 & $5.48 \mathrm{E}-12$ & 0.99997 & $2.43 \mathrm{E}-12$ & 0.99998 & $1.15 \mathrm{E}-12$ & 0.99998 & $5.62 \mathrm{E}-13$ & 0.99998 \\
\hline 30 & $3.90 \mathrm{E}-12$ & 0.99998 & $1.73 \mathrm{E}-12$ & 0.99998 & $8.22 \mathrm{E}-13$ & 0.99998 & $4.00 \mathrm{E}-13$ & 0.99998 \\
\hline
\end{tabular}

020001-12 


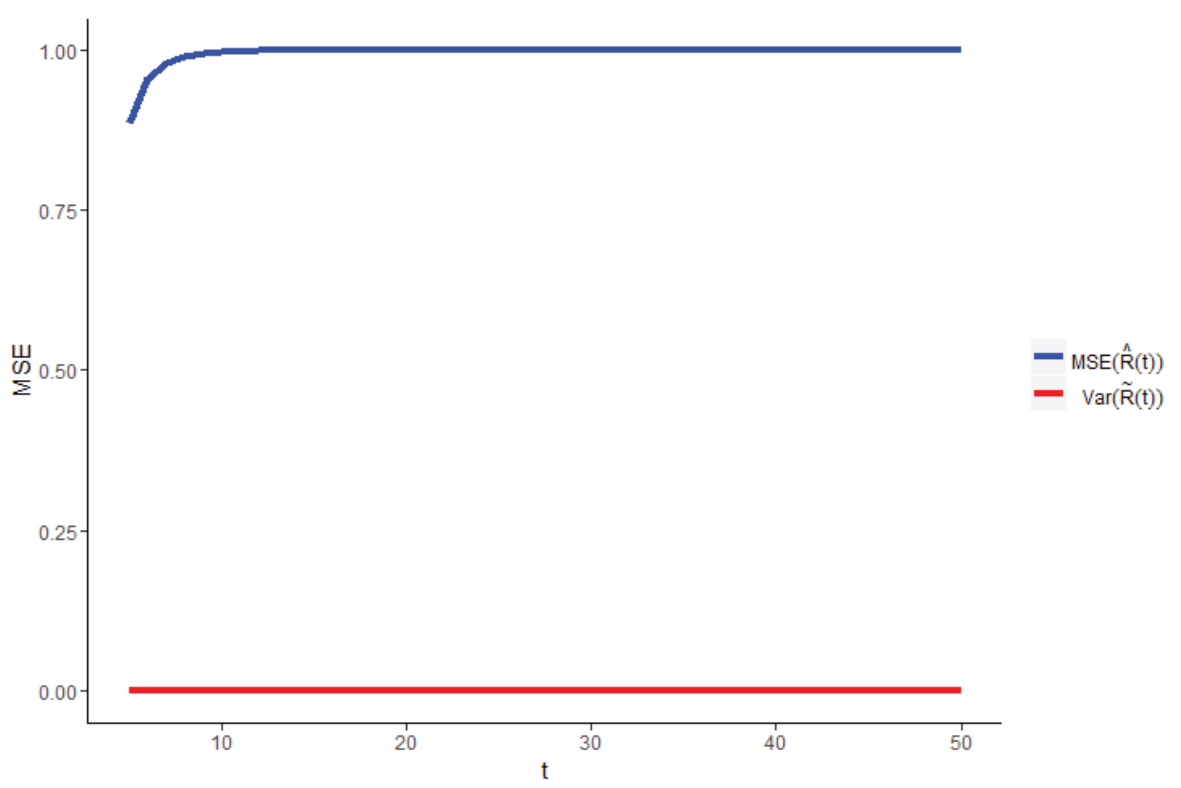

FIGURE 2. Mean Square Error of MLE and UMVUE of Reliability function for $n=20$.

Figure 3 shows the $p d f$ plot of $\operatorname{GIWD}(\alpha, \beta, \gamma)$ for $\alpha=3, \beta=2$ and $\gamma=2$ and also displays the MLE and UMVUE of sampled $p d f$.

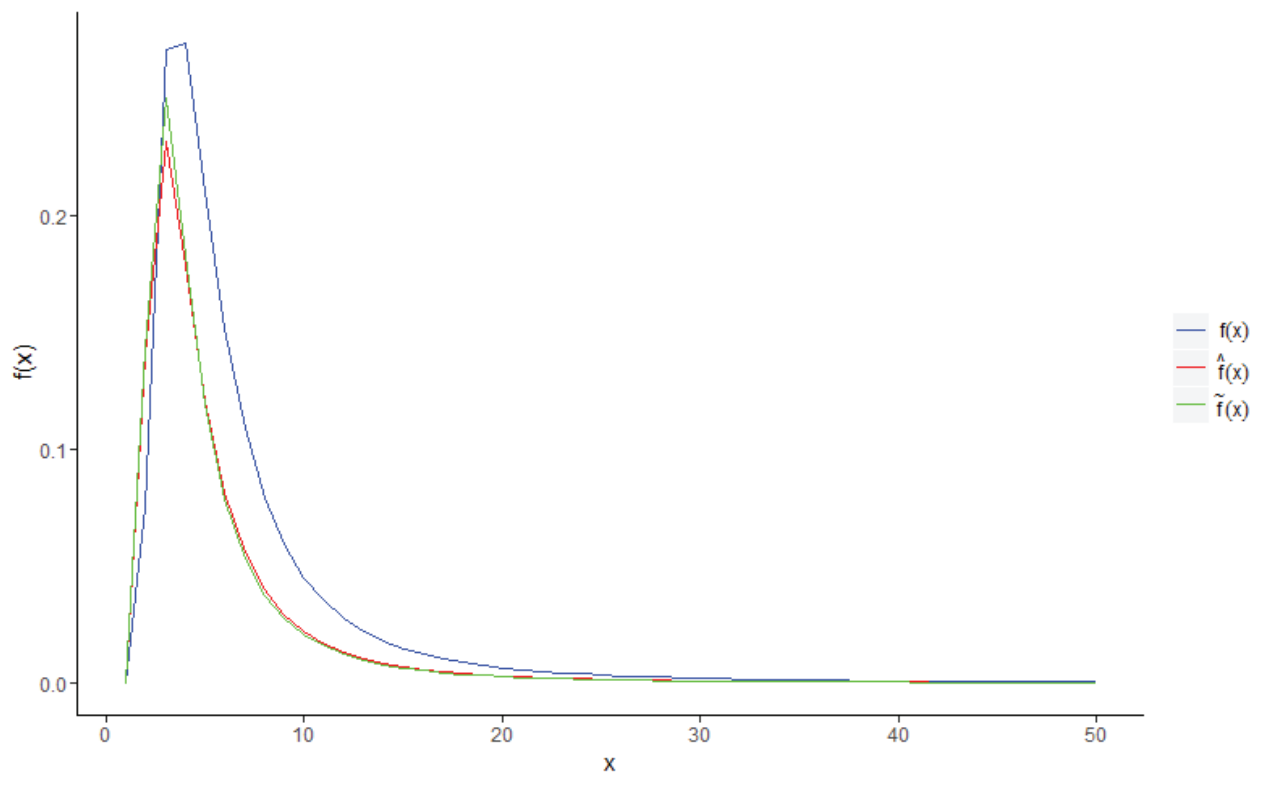

FIGURE 3. MLE and UMVUE of sampled $p d f$. 
TABLE 3. CI and length of CI based on MLE and log transformed MLE of $\alpha$ and $\beta$ at $95 \%$ and $90 \%$ level of significance.

\begin{tabular}{ccccccccc}
\hline$n$ & & $\alpha$ & \multicolumn{2}{c}{$\log (\alpha)$} & \multicolumn{2}{c}{$\log (\beta)$} \\
& $95 \%$ & $90 \%$ & $95 \%$ & $90 \%$ & $95 \%$ & $90 \%$ & $95 \%$ & $90 \%$ \\
\hline \multirow{2}{*}{5} & {$[0.4339$} & {$[0.5249$} & {$[0.5677$} & {$[0.6218$} & {$[0.3994$} & {$[0.6567$} & {$[0.8983$} & {$[1.0217$} \\
& $1.5660]$ & $1.4750]$ & $1.7612]$ & $1.6080]$ & $3.6005]$ & $3.3432]$ & $4.4524]$ & $3.9148]$ \\
& 1.1320 & 0.9500 & 1.1935 & 0.9862 & 3.2011 & 2.6865 & 3.5540 & 2.8931 \\
\hline \multirow{2}{*}{10} & {$[0.5814$} & {$[0.6487$} & {$[0.6579$} & {$[0.7037$} & {$[0.8172$} & {$[1.0074$} & {$[1.1071$} & {$[1.2175$} \\
& $1.4185]$ & $1.3512]$ & $1.5198]$ & $1.4208]$ & $3.1827]$ & $2.9925]$ & $3.6128]$ & $3.2852]$ \\
& 0.8372 & 0.7026 & 0.8618 & 0.7171 & 2.3654 & 1.9851 & 2.5057 & 2.0676 \\
\hline \multirow{2}{*}{15} & {$[0.6535$} & {$[0.7092$} & {$[0.7071$} & {$[0.7476$} & {$[1.0200$} & {$[1.1775$} & {$[1.2252$} & {$[1.3256$} \\
& $1.3464]$ & $1.2907]$ & $1.4140]$ & $1.3374]$ & $2.9799]$ & $2.8224]$ & $3.2646]$ & $3.0173]$ \\
& 0.6929 & 0.5815 & 0.7069 & 0.5897 & 1.9599 & 1.6448 & 2.0393 & 1.6916 \\
\hline \multirow{2}{*}{20} & {$[0.6900$} & {$[0.7399$} & {$[0.7335$} & {$[0.7709$} & {$[1.1234$} & {$[1.2643$} & {$[1.2903$} & {$[1.3845$} \\
& $1.3099]$ & $1.2600]$ & $1.3632]$ & $1.2970]$ & $2.8765]$ & $2.7356]$ & $3.1000]$ & $2.8891]$ \\
& 0.6198 & 0.5201 & 0.6297 & 0.5260 & 1.7530 & 1.4712 & 1.8097 & 1.5046 \\
\hline
\end{tabular}

TABLE 4. CI and length of CI based on MLE and log transformed MLE of $\gamma$ and CI and length of CI of $R(t)$ at $95 \%$ and $90 \%$ level of significance.

\begin{tabular}{cccccccc}
\hline$n$ & \multicolumn{2}{c}{$\gamma$} & \multicolumn{2}{c}{$\log (\gamma)$} & \multicolumn{2}{c}{$R(t)$} \\
& $95 \%$ & $90 \%$ & $95 \%$ & $90 \%$ & $95 \%$ & $90 \%$ \\
\hline 5 & {$[0.79937 .2006]$} & {$[1.31396 .6860]$} & {$[1.79708 .9035]$} & {$[2.04377 .8287]$} & {$[0.10430 .2600]$} & {$[0.10670 .2584]$} \\
& 6.4012 & 5.3720 & 7.1064 & 5.7850 & 0.1557 & 0.1517 \\
\hline 10 & {$[1.63616 .3638]$} & {$[2.01625 .9837]$} & {$[2.21527 .2228]$} & {$[2.43596 .5681]$} & {$[0.10900 .2456]$} & {$[0.11240 .2271]$} \\
& 4.7276 & 3.9675 & 5.0076 & 4.1321 & 0.1366 & 0.1146 \\
\hline 15 & {$[2.04005 .9599]$} & {$[2.35515 .6448]$} & {$[2.45056 .5292]$} & {$[2.65136 .0345]$} & {$[0.11260 .2258]$} & {$[0.11610 .2103]$} \\
& 3.9199 & 3.2897 & 4.0786 & 3.3832 & 0.1132 & 0.0941 \\
\hline 20 & {$[2.24695 .7530]$} & {$[2.52875 .4712]$} & {$[2.58066 .2000]$} & {$[2.76905 .7782]$} & {$[0.11480 .2156]$} & {$[0.11840 .2021]$} \\
& 3.5060 & 2.9424 & 3.6194 & 3.0091 & 0.1007 & 0.0836 \\
\hline
\end{tabular}

For computations shown in Table 3 and Table 4 , we have considered $\alpha=1, \beta=2$ and $\gamma=3$. For $t=$ $5, R(t)=0.1478$. From Table 3 and Table 4 we observe that as sample size increases, the length of CIs based on MLE and log-transformed MLE decreases. As reported by Meeker and Escober we too observe that asymptotic CIs based on log-transformed MLE have better coverage probability.

For computations shown in Table 5 and Table 6 , we have considered $\alpha=3, \beta=2$ and $\gamma=7$. For $t=$ $5, R(t)=0.9195$ and we compute point estimate and interval estimate (CI) of UMVUE and MLE of $\gamma$ and $R(t)$. From Table 5 and Table 6 we observe that as sample size increases, the length of CI of UMVUE and MLE of $\gamma$ and $R(t)$ decreases. 
TABLE 5. Point estimate, Interval estimate and length of CI of UMVUE and MLE of $\gamma$ at $95 \%$ and $90 \%$ level of significance.

\begin{tabular}{cccccccc}
\hline$n$ & $\tilde{\gamma}$ & \multicolumn{3}{c}{$\tilde{\gamma}$} & $\hat{\gamma}$ & \multicolumn{2}{c}{$\hat{\gamma}$} \\
& & $95 \%$ & $90 \%$ & $95 \%$ & $90 \%$ \\
\hline 5 & 5.6523 & {$[2.999515 .8954]$} & {$[3.329213 .3944]$} & 6.7828 & {$[3.599419 .0744]$} & {$[3.995016 .0733]$} \\
& & 12.8958 & 10.0652 & & 15.4750 & 12.0783 \\
10 & 5.6819 & {$[3.806312 .7477]$} & {$[4.126811 .3470]$} & 6.2501 & {$[4.186914 .0225]$} & {$[4.539512 .4817]$} \\
& & 8.9414 & 7.2202 & & 9.8355 & 7.9422 \\
15 & 6.5640 & {$[4.244111 .4812]$} & {$[4.546010 .4623]$} & 7.0016 & {$[4.527012 .2466]$} & {$[4.849011 .1598]$} \\
& & 7.2371 & 5.9163 & & 7.7195 & 6.3107 \\
20 & 7.0067 & {$[4.532410 .7697]$} & {$[4.81729 .9488]$} & 7.3571 & {$[4.759011 .3082]$} & {$[5.058110 .4462]$} \\
& & 6.2373 & 5.1315 & & 6.5492 & 5.3881 \\
\hline
\end{tabular}

TABLE 6. Point estimate, Interval estimate and length of CI of UMVUE and MLE of $R(t)$ at $95 \%$ and $90 \%$ level of significance.

\begin{tabular}{|c|c|c|c|c|c|c|}
\hline \multirow[t]{2}{*}{$n$} & \multirow{2}{*}{$\tilde{R}(t)$} & \multicolumn{2}{|c|}{$\tilde{R}(t)$} & \multirow{2}{*}{$\hat{R}(t)$} & \multicolumn{2}{|c|}{$\hat{R}(t)$} \\
\hline & & $95 \%$ & $90 \%$ & & $95 \%$ & $90 \%$ \\
\hline \multirow[t]{2}{*}{5} & 0.9266 & {$\left[\begin{array}{lll}0.7037 & 1.0000\end{array}\right]$} & {$\left[\begin{array}{lll}0.7459 & 0.9999\end{array}\right]$} & 0.9129 & {$\left[\begin{array}{lll}0.7263 & 0.9989\end{array}\right]$} & {$\left[\begin{array}{lll}0.7626 & 0.9969\end{array}\right]$} \\
\hline & & 0.2963 & 0.2540 & & 0.2726 & 0.2342 \\
\hline \multirow[t]{2}{*}{10} & 0.8985 & {$\left[\begin{array}{lll}0.7709 & 0.9978\end{array}\right]$} & {$\left[\begin{array}{lll}0.7997 & 0.9947\end{array}\right]$} & 0.8946 & {$\left[\begin{array}{lll}0.7784 & 0.9935\end{array}\right]$} & {$\left[\begin{array}{lll}0.8048 & 0.9888\end{array}\right]$} \\
\hline & & 0.2269 & 0.1949 & & 0.2150 & 0.1839 \\
\hline \multirow[t]{2}{*}{15} & 0.9235 & {$\left[\begin{array}{lll}0.8003 & 0.9920\end{array}\right]$} & {$\left[\begin{array}{ll}0.8232 & 0.9869\end{array}\right]$} & 0.9195 & {$\left[\begin{array}{lll}0.8040 & 0.9878\end{array}\right]$} & {$\left[\begin{array}{lll}0.8254 & 0.9820\end{array}\right]$} \\
\hline & & 0.1916 & 0.1636 & & 0.1838 & 0.1565 \\
\hline \multirow[t]{2}{*}{20} & 0.9325 & {$\left[\begin{array}{lll}0.8176 & 0.9865\end{array}\right]$} & {$\left[\begin{array}{lll}0.8370 & 0.9806\end{array}\right]$} & 0.9292 & {$\left[\begin{array}{lll}0.8197 & 0.9829\end{array}\right]$} & {$\left[\begin{array}{lll}0.8381 & 0.9767\end{array}\right]$} \\
\hline & & 0.1688 & 0.1436 & & 0.1632 & 0.1386 \\
\hline
\end{tabular}

In the theory developed in Section 5, we have considered record values from $\operatorname{GIWD}(\alpha, \beta, \gamma)$ for $\alpha=10$, $\beta=2$ and $\gamma=5$.

$\begin{array}{lllllllll}19.63889 & 23.03414 & 24.51706 & 50.14350 & 198.77064 & 312.60405 & 328.34775 & 857.29468 & 1397.77133\end{array}$ $9088.19426 \quad 13213.86061$

The MLE and UMVUE of $\gamma$ are obtained as $\hat{\gamma}=5.8045$ and $\tilde{\gamma}=5.2768$. For testing the hypothesis $H_{\mathrm{o}}: \gamma=$ $\gamma_{\mathrm{o}}=5.8$ against $H_{1}: \gamma \neq \gamma_{\mathrm{o}}=5.8$ under this scheme, with the help of Chi-Square tables at $5 \%$ level of significance, we obtained $l_{\mathrm{o}}=0.9460$ and $l_{\mathrm{o}}{ }^{\prime}=3.1682$. Hence, in this case we may accept $H_{\mathrm{o}}$ at $5 \%$ level of significance since $U\left(R_{n}\right)=1.8950$. Again, for testing $H_{\mathrm{o}}: \gamma \leq \gamma_{\mathrm{o}}=5.8$ against $H_{1}: \gamma>\gamma_{\mathrm{o}}=5.8$, we obtained $l_{\mathrm{o}}{ }^{\prime \prime}=1.0627$ and hence, in this case we may accept $H_{\mathrm{o}}$ at 5\% level of significance. Now, for testing the hypothesis $H_{\mathrm{o}}: \gamma=\gamma_{\mathrm{o}}=5.2$ against $H_{1}: \gamma \neq \gamma_{\mathrm{o}}=5.2$ under this scheme, with the help of Chi-Square tables at 5\% level of significance, we obtained $l_{\mathrm{o}}=1.0406$ and $l_{\mathrm{o}}{ }^{\prime}=3.4850$. Hence, in this case we may accept $H_{\mathrm{o}}$ at $5 \%$ level of significance since $U\left(R_{n}\right)=1.8950$. Again, for testing $H_{\mathrm{o}}: \gamma \leq \gamma_{\mathrm{o}}=5.2$ against $H_{1}: \gamma>\gamma_{\mathrm{o}}=5.2$, we obtained $l_{\mathrm{o}}{ }^{\prime \prime}=1.1690$ and hence, in this case we may accept $H_{\mathrm{o}}$ at $5 \%$ level of significance. 


\section{ACKNOWLEDGMENTS}

The authors are grateful to the reviewers for their valuable comments.

\section{REFERENCES}

1. Arnold, B. C., Balakrishan, N. and Nagaraja, H. N., A First Course in Order Statistics (John Wiley \& Sons, New York, 1992).

2. Arashi, M. and Emadi, M., Stat. Papers 13(8), 380-210 (2008).

3. Awad, A. M. and Gharraf, M. K., Commun. Statist. - Simul. 15(2), 389-403 (1986).

4. Balakrishan, N., Ahsanullah, M. and Chan, P. S., J. App. Statist. Scien. 2, 233-248 (1995).

5. Bartholomew, D. J., J. Amer. Statist. Assoc. 52, 350-355 (1957).

6. Bartholomew, D. J., Technometric 5(3), 361-374 (1963).

7. Basu, A. P., Technometrics 6, 215-219 (1964).

8. Belaghi, R. A., Arashi, M. and Tabatabaey, S. M. M., "On the Construction of Preliminary Test Estimator Based on Record Values for the Burr XII Model", Commun. Statist. - Theo. Meth. 44(1), 1-23 (2015).

9. Constantine, K., Karson, M. and Tse, S. K., "Estimation of $\mathrm{P}(\mathrm{Y}<\mathrm{X})$ in the gamma case", Commun. Statist. Simul. 15(2), 365-388 (1986).

10. Chandler, K. N., J. Royal Statist. Socie. Series B 14, 220-228 (1952).

11. Chao, A., IEEE Trans. Reliability R-26, 389-392 (1982).

12. Chaturvedi, A. and Kumari, T., "Estimation and testing procedures for the reliability functions of a family of lifetime distributions", interstat.statjournals.net/ YEAR/ 2015/ abstracts/ 1306001.php (2015).

13. Chaturvedi, A. and Malhotra, A., Int. J. Syst. Assur. Eng. Manag. DOI:10.1007/s13198-016-0531-2 (2016).

14. Chaturvedi, A. and Malhotra, A., Appl. Math. Inf. Scien. 11(3), 837-849 (2017).

15. Chaturvedi, A., Pathak, A., J. Stat. \& Appl. 7, 1-8 (2012).

16. Chaturvedi, A. and Pathak, A., "Bayesian estimation procedures for three parameter exponentiated Weibull distribution under entropy loss function and type II censoring", interstat. statjournals. net/YEAR/2013/abstracts/1306001.php (2013).

17. Chaturvedi, A. and Pathak, A., IJSER 5(1), 1171-1180 (2014).

18. Chaturvedi, A. and Rani, U., Metrika 46, 213-219 (1997).

19. Chaturvedi, A. and Rani, U., J. Statist. Res. 32, 113-120 (1998).

20. Chaturvedi, A. and Singh, K. G., Metron 64(2), 179-198 (2006).

21. Chaturvedi, A. and Singh, K. G., J. Appl. Statist. Sci. 16(2), 35-50 (2008).

22. Chaturvedi, A. and Surinder, K., Brazilian J. Prob. Statist. 13, 29-39 (1999).

23. Chaturvedi. A. and Tomer, S. K., J. App. Statist. Scien. 11, 33-43 (2002).

24. Chaturvedi, A. and Tomer, S. K., Statist. Papers 44(3), 301-313 (2003).

25. Erdelyi, A.(Ed)., Tables of Integral Transforms (McGraw-Hill, 1, 1954).

26. Glick, N., Amer. Mathe. Month. 85, 543-551 (1978).

27. Gusmão, F. R. S. de., Ortega, E. M. M. and Cordeiro, G. M., Statist. Papers, DOI: 10.1007/s00362-009-0271-3 (2009).

28. Habibi Rad, A., Arghami, N. R. and Ahmadi, J., Commun. Stat. Theo. Meth. 35(11), 1971-1983 (2006).

29. Johnson, N. L., Technometrics 17, 393 (1975).

30. Keller, A. Z. and Kamath, A. R., "Reliability analysis of CNC machine tools", Reliab. Eng. 3, 449-473 (1982).

31. Kelly, G. D., Kelly, J. A. and Schucany, W. R., "Efficient estimation of $\mathrm{P}(\mathrm{Y}<\mathrm{X})$ in the exponential case", Technometrics 18, 359-360 (1976).

32. Lehmann, E. L., Testing Statistical Hypotheses (John Wiley and Sons, New York, 1959).

33. Meeker, W. Q. and Escober, L. A., Statistical Methods for Reliability Data (John Wiley and Sons, New York, 1998).

34. Nagaraja, H. N., "Record values and related statistics - A review", Commun. Statist. Theo. Meth. 17, 22232238 (1988a).

35. Nagaraja, H. N., "Some characterizations of continuous distributions based on regressions of adjacent order statistics and record values", Sankhya, Series A 50, 70-73 (1988b).

36. Pugh, E. L., "The best estimate of reliability in the exponential case", Operations Research 11, 57-61 (1963). 
37. Razmkhah, M. and Ahmadi, J., "Comparing two sampling schemes based on entropy of record statistics", Statist. Papers 53, 95-106 (2011).

38. Rohtagi, V. K. and Saleh, Eh. A. K. Md., An Introduction to Probability and Statistics, Second Edition (John Wiley \& Sons, U.K, 2012).

39. Sathe, Y. S. and Shah, S. P., "On estimating $\mathrm{P}(\mathrm{X}<\mathrm{Y})$ for the exponential distribution", Commun. Statist. Theo. Meth. A10, 39-47 (1981).

40. Tong, H., "A note on the estimation of $\mathrm{P}(\mathrm{Y}<\mathrm{X})$ in the exponential case", Technometrics 16, 625 (1974).

41. Tong, H., "Letter to the editor", Technometrics 17, 393 (1975).

42. Tyagi, R. K. and Bhattacharya, S. K., "A note on the MVU estimation of reliability for the Maxwell failure distribution", Estadistica 41, 73-79 (1989).

43. Watson, R. I., "Research design and methodology in evaluating the results of psychotherapy", J. Clin. Psychol. 8, 29-33 (1952). 